\title{
1/f-noise study of undoped intrinsic hydrogenated amorphous silicon thin films
}

\author{
Mehmet Güneş, ${ }^{*}$ Robert E. Johanson, and S. O. Kasap \\ Department of Electrical Engineering, University of Saskatchewan, Saskatoon, SK, Canada S7N 5A9
}

(Received 28 January 1999)

\begin{abstract}
Conductance fluctuations in four samples of undoped intrinsic hydrogenated amorphous silicon $(a-\mathrm{Si}: \mathrm{H})$ were measured in the temperature range of $450 \mathrm{~K}$ to $500 \mathrm{~K}$ and for frequencies from $2 \mathrm{~Hz}$ to $3 \mathrm{kHz}$. The noise spectra divide into two regions that each fit a $1 / f^{\alpha}$ power law but with different slope parameters $\alpha$ and different temperature dependences. At low frequencies, $\alpha$ is greater than unity and increases with temperature. At high frequencies, $\alpha$ is near 0.6 and temperature independent, but the noise magnitude decreases rapidly with temperature. We infer from the different dependences on temperature that the noise is generated by two independent mechanisms operating simultaneously in $a$-Si:H. We also observe that the $1 / f$ noise exhibits a quadratic dependence on bias current and Gaussian statistics. [S0163-1829(99)04327-1]
\end{abstract}

Experimental studies of conductance fluctuations in $n$-type hydrogenated amorphous silicon $(a-\mathrm{Si}: \mathrm{H})$ have found that the noise power density spectrum follows the usual $1 / f^{\alpha}$ power law (with $\alpha$ near unity) that is typical of most conducting materials. ${ }^{1-5}$ Some of these studies report unexpected properties of the noise such as a nonquadratic dependence on bias current and non-Gaussian statistics, $3,5,6$ although other studies do not find these unusual features. ${ }^{4,7,8}$ There have been fewer studies of noise in undoped $a-\mathrm{Si}: \mathrm{H}$, perhaps because the high room-temperature resistivity requires either a transverse geometry which often leads to nonOhmic behavior or high temperatures. ${ }^{5,6,9,10}$ A recent publication reports on $1 / f$ noise in undoped, but slightly $n$-type, $a$-Si:H films, with conductivity activation energy $E_{\sigma}$ of 0.4 $\mathrm{eV}$ and $0.80 \mathrm{eV}$ deposited by radio-frequency (RF) plasmaenhanced chemical-vapor deposition (PECVD), and hot-wire CVD, respectively. ${ }^{6}$ In these samples, the noise power has the expected quadratic dependence on the bias current, but, as in the doped material, strongly non-Gaussian statistics. Others studying PECVD material find Gaussian behavior. ${ }^{10}$ Another paper reports that $\alpha$ varies with temperature increasing from 0.7 at $328 \mathrm{~K}$ to 1.1 at $428 \mathrm{~K}$. ${ }^{9}$ Undoped and doped $a$-Si:H differ not only in the position of the Fermi level but also in the density and charge state of defects and the hydrogen diffusion coefficient. Motivated by a desire to test whether these differences influence the level or spectrum of noise, we conducted noise measurements of intrinsic $a-\mathrm{Si}: \mathrm{H}$.

Thin films of device quality, undoped intrinsic $a-\mathrm{Si}: \mathrm{H}$ were fabricated in a dc glow-discharge deposition system at Solarex using conditions optimized to produce a low defect density. ${ }^{11}$ Coplanar $\mathrm{NiCr}$ electrodes were evaporated onto the Corning 7059 glass substrates before the deposition. All measurements used two-probe techniques. We chose a coplanar geometry because sandwich structures often display nonlinear current voltage (I-V) curves. In our samples, the I-V relation is linear to the highest currents used for noise measurements (see inset of Fig. 2). The four samples studied here were previously characterized in detail by subbandgap optical absorption and steady-state photoconductivity measurements. $^{12,13}$ Various properties are summarized in Table I. The samples differ in deposition temperature which primarily causes variation in the band gap. Noise and conductivity measurements were carried out under 1 Torr of flowing ultrahigh purity (uhp) helium to reduce the effects of surface contaminants. Prior to the noise measurements, the samples were annealed for up to $2 \mathrm{~h}$ at the highest measurement temperature. The temperature was kept below the deposition temperature to avoid nonreversible structural changes. Because the conductivity of intrinsic $a-\mathrm{Si}: \mathrm{H}$ has a large activation energy, noise data could be obtained only over the temperature range of $450 \mathrm{~K}$ to $505 \mathrm{~K}$; the resistance below $450 \mathrm{~K}$ was too large to allow noise measurements.

The apparatus and procedures used to acquire noise spectra were described in detail previously. ${ }^{4}$ At each temperature, noise spectra were obtained for several dc bias currents ranging from 0.5 to $20 \mu \mathrm{A}$. The current density was kept below $0.3 \mathrm{~A} / \mathrm{cm}^{2}$ to avoid self-heating. The background noise which consists of Johnson and instrumental noise was measured separately and subtracted leaving only the noise due to conductance fluctuations.

Figure 1 shows the noise power density $S_{n}$ versus frequency for sample $\mathrm{S} 260$ at $452 \mathrm{~K}$ for four bias currents from $1 \mu \mathrm{A}$ to $8 \mu \mathrm{A}$. The data can not be fit to a single $1 / f^{\alpha}$ power law but rather show two distinct regions that separately fit a power law. At low frequencies, which we call region 1, $\alpha_{1}$ $=1.20$, and at high frequencies, region $2, \alpha_{2}=0.58$. As shown in the inset to Fig. $1, S_{n}$ varies with bias current as $I^{b}$ with $b=2.0 \pm 0.04$. To be certain that the unusual shape of the noise spectrum is not an artifact caused by temperature fluctuations due to turbulence in the helium atmosphere, we

TABLE I. Physical, electrical, and optical properties of the four $a$-Si:H studied where $E_{\sigma}$ is the conductivity activation energy, $E_{04}$ is the band gap, $d$ is the thickness, $\alpha_{1.2}$ is the optical absorption coefficient at $1.2 \mathrm{eV}$, and $N_{d}$ is the defect density.

\begin{tabular}{ccccccc}
\hline \hline Sample & $\begin{array}{c}E_{\sigma} \\
(\mathrm{eV})\end{array}$ & $\begin{array}{c}E_{04} \\
(\mathrm{eV})\end{array}$ & $\begin{array}{c}d \\
(\mu \mathrm{m})\end{array}$ & $\begin{array}{c}\text { Volume } \\
\left(\mathrm{cm}^{-3}\right)\end{array}$ & $\begin{array}{c}\alpha_{1.2} \\
\left(\mathrm{~cm}^{-1}\right)\end{array}$ & $\begin{array}{c}N_{d} \\
\left(\mathrm{~cm}^{-3}\right)\end{array}$ \\
\hline S260 & 0.94 & 1.90 & 1.0 & $2.4 \times 10^{-6}$ & 0.23 & $7 \times 10^{15}$ \\
S215 & 1.04 & 2.01 & 1.45 & $6.3 \times 10^{-6}$ & 0.13 & $4 \times 10^{15}$ \\
S300 & 0.88 & 1.86 & 1.01 & $2.2 \times 10^{-5}$ & 0.18 & $6 \times 10^{15}$ \\
MBA1 & 0.94 & 1.90 & 0.98 & $2.9 \times 10^{-6}$ & 0.20 & $6 \times 10^{15}$ \\
\hline \hline
\end{tabular}




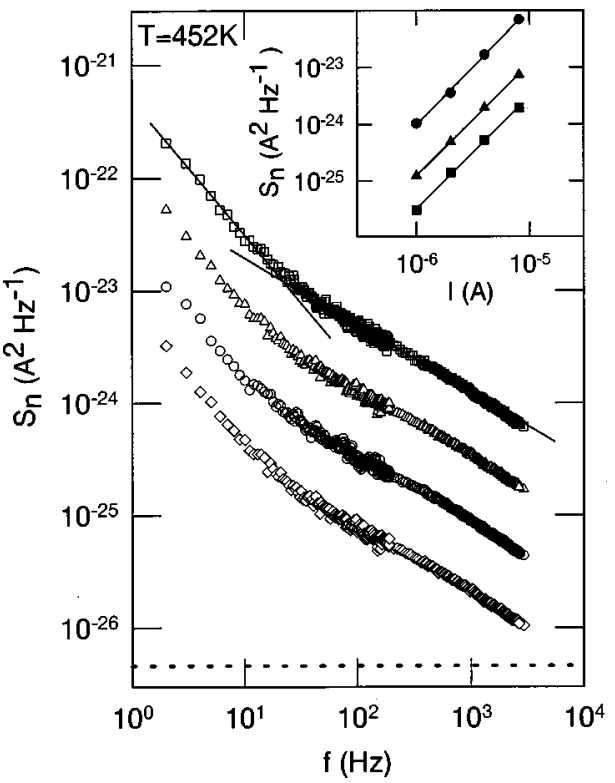

FIG. 1. The noise power density spectra of intrinsic $a-\mathrm{Si}: \mathrm{H}$ (sample S260) for bias currents of 8, 4, 2, and $1 \mu \mathrm{A}$, top to bottom, at $452 \mathrm{~K}$. The dashed line indicates the level of Johnson noise. Inset: the noise power density versus dc bias current at $5 \mathrm{~Hz}$ (circles), $50 \mathrm{~Hz}$ (triangles), and $500 \mathrm{~Hz}$ (squares).

added a copper cover to the heating stage, completely enclosing the sample. Any turbulence around the sample should have been greatly reduced yet no change in the spectrum was observed.

Noise spectra for the same sample at $505 \mathrm{~K}$ are plotted in Fig. 2, again for four different bias currents. Here the spectra can be fitted to a single power law with $\alpha_{1}=1.30$ for all but the highest frequencies; the small upward curvature is all that remains of region 2. The dominance of region 1 is caused

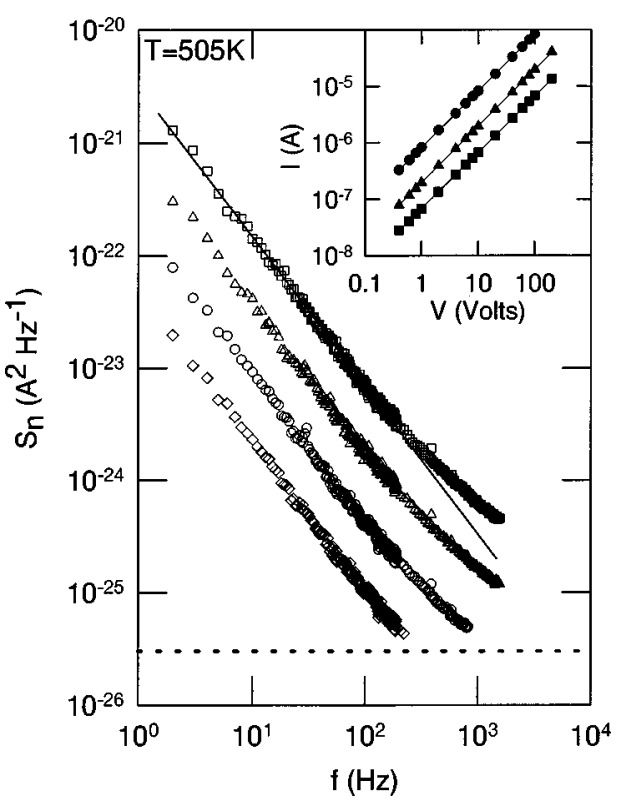

FIG. 2. The noise power density spectra of intrinsic $a-\mathrm{Si}: \mathrm{H}$ (sample S260) for bias currents of 16, 8, 4, and $2 \mu \mathrm{A}$, top to bottom, at $505 \mathrm{~K}$. The dashed line indicates the level of Johnson noise. Inset: the current-voltage relationship at three temperatures; circles $505 \mathrm{~K}$, triangles $471 \mathrm{~K}$, and squares $452 \mathrm{~K}$.
TABLE II. Summary of the fitting parameter to the noise spectra at various temperatures for each sample.

\begin{tabular}{ccccc}
\hline \hline T $(\mathrm{K})$ & $R(M \Omega)$ & $\alpha_{1}$ & $\alpha_{2}$ & $f_{\text {kink }}(\mathrm{Hz})$ \\
\hline \multicolumn{5}{c}{ Sample S260 } \\
452 & 16 & 1.21 & 0.58 & 20 \\
471 & 5.1 & 1.25 & 0.58 & 60 \\
505 & 1.2 & 1.30 & & 500 \\
& \multicolumn{5}{c}{ Sample S215 } & \\
454 & 16 & 1.14 & 0.56 & 30 \\
467 & 9.1 & 1.19 & 0.55 & 100 \\
482 & 5.0 & 1.27 & 0.57 & 200 \\
& & Sample S300 & \\
450 & 20 & 1.03 & 0.62 & 20 \\
472 & 8.3 & 1.11 & 0.64 & 100 \\
493 & 3.4 & 1.16 & & 400 \\
454 & 10 & Sample MBA1 & \\
468 & 5.8 & 1.14 & 0.59 & 30 \\
495 & 1.6 & 1.28 & 0.59 & 80 \\
\hline \hline
\end{tabular}

either by an increase in the noise magnitude at low frequencies or a decrease in the magnitude at high frequencies. In order to compare the magnitudes at different temperatures it is important to compare curves obtained with the same bias current. Focusing on the curves for $8 \mu \mathrm{A}$, we see that, in spite of the small increase in $\alpha_{1}$, the magnitude of the noise in region 1 is roughly the same at $452 \mathrm{~K}$ and $505 \mathrm{~K}$. In contrast, the excess noise at high frequencies has decreased by at least an order of magnitude. Examining spectra from intermediate temperatures shows that the curve in region 2 shifts uniformly downward with temperature but maintains the slope parameter $\alpha_{2}$ near 0.6.

Similar results were obtained for the three other intrinsic $a-\mathrm{Si}: \mathrm{H}$ films studied. Results for all samples are summarized in Table II. In each case, the noise spectra at lower temperatures display two distinct power law regions characterized by $\alpha_{1}$ and $\alpha_{2} . \alpha_{1}$ has more sample to sample variation but is typically greater than unity and increases with temperature. $\alpha_{2}$ is near 0.6 for all the samples and does not vary with temperature. However, for all samples, the magnitude of the noise in region 2 decreases rapidly with increasing temperature, resulting in the crossover point, labeled $f_{\text {kink }}$ in the table, moving to higher frequency.

The statistics of the noise signal were also investigated by measuring the correlation in fluctuations of noise power density at a set of discrete frequencies. The method is described in Ref. 7. Correlation experiments were run on several of the $a$-Si:H samples at several temperatures. In all cases, the histogram of cross-correlation coefficients is centered about zero to \pm 0.01 with a shape consistent with that expected for an uncorrelated signal.

The natural interpretation of these results is that two separate noise generating mechanisms are active in undoped, intrinsic $a$-Si:H. One mechanism produces a frequency distribution with $\alpha$ greater than unity and the other with $\alpha$ near 0.6. The different slopes mean that the former is dominant at low frequencies and the latter at high frequencies. We were fortunate that in the temperature range examined the cross- 
over point $f_{\text {kink }}$ occurred in the frequency range of our equipment. This is, to our knowledge, the first direct experimental evidence that two noise processes contribute to Gaussian $1 / f$-type noise in $a$-Si:H.

We note that the noise data reported here differ from some of the previously reported results for undoped $a-\mathrm{Si}: \mathrm{H}$ films. Khera et al. measured the second spectrum and found strongly non-Gaussian statistics. ${ }^{5,6}$ However, the $a$-Si:H used in that study, although undoped, was not intrinsic but slightly $n$-type. Bathai and Anderson studied intrinsic $a-\mathrm{Si}: \mathrm{H}$ but did not observe deviations from a simple power law in the noise spectrum. ${ }^{9}$ Recently, however, Reynolds et al. reported a noise spectrum with excess weight at higher frequencies in intrinsic $a$-Si:H similar to that reported here. ${ }^{14}$ However, data at only one temperature were reported. They also found the noise is Gaussian. These discrepancies might simply reflect sample to sample variation. Even device quality, undoped $a$-Si:H varies widely in, for example, optoelectronic properties. ${ }^{15}$ Undoped intrinsic $a-\mathrm{Si}: \mathrm{H}$ (with $E_{\sigma}$ of $0.90 \mathrm{eV}$ ) also differs from undoped nonintrinsic $a-\mathrm{Si}: \mathrm{H}$ (with $E_{\sigma}$ from $0.6 \mathrm{eV}$ to $0.80 \mathrm{eV}$ ) in that the former contains fewer charged defects. ${ }^{13}$ Possibly, there exist a number of noise sources in $a-\mathrm{Si}: \mathrm{H}$, and a different source dominates depending on the particular properties of the sample and the conditions of measurement. To test this idea, further work on $1 / f$ noise in different undoped intrinsic and nonintrinsic $a$-Si:H films deposited in different systems is underway and will be reported in the near future.

We thank NSERC for providing financial support, and we are grateful to Professor C. Wronski for providing the samples.
*Permanent address: Department of Physics, Izmir Institute of Technology, Gaziosmanpaşa Bulv. No:16, Çankaya, Izmir, 35210, Turkey.

${ }^{1}$ J. C. Anderson, Philos. Mag. B 48, 31 (1983).

${ }^{2}$ A. D'Amico, G. Fortunato, and C. M. V. Vliet, Solid-State Electron. 28, 837 (1985)

${ }^{3}$ C. E. Parman, N. E. Israeloff, and J. Kakalios, Phys. Rev. B 47, 12578 (1993).

${ }^{4}$ R. E. Johanson, D. Scansen, and S. O. Kasap, Philos. Mag. B 73, 707 (1996).

${ }^{5}$ G. M. Khera and J. Kakalios, Phys. Rev. B 56, 1918 (1997).

${ }^{6}$ G. M. Khera, J. Kakalios, and E. Iwaniczko, Mater. Res. Soc. Symp. Proc. 420, 641 (1996).

${ }^{7}$ R. E. Johanson, D. Scansen, and S. O. Kasap, J. Vac. Sci. Technol. B 17, 73 (1999).

${ }^{8}$ M. Güneş, R. E. Johanson, and S. O. Kasap, in Proceedings of the 10th International School on Condensed Matter Physics, edited by J. M. Marshall (World Scientific, Singapore, in press).

${ }^{9}$ F. Z. Bathei and J. C. Anderson, Philos. Mag. B 55, 87 (1987).

${ }^{10}$ P. A. W. E. Verleg and J. I. Dijkhuis, Phys. Rev. B 58, 3904 (1998).

${ }^{11}$ C. M. Fortmann, J. O'Dowd, N. Newton, and J. Fisher, in Stability of Amorphous Silicon Alloy Materials and Devices, edited by B. L. Stafford and E. Sabisky, AIP Conf. Proc. 157 (AIP, New York, 1987), p. 103.

${ }^{12}$ M. Güneş, R. W. Collins, and C. R. Wronski, Mater. Res. Soc. Symp. Proc. 336, 413 (1994).

${ }^{13}$ M. Güneş and C. R. Wronski, J. Appl. Phys. 81, 3526 (1997).

${ }^{14}$ S. Reynolds, C. Main, and M. J. Rose, J. Non-Cryst. Solids 227230, 233 (1998).

${ }^{15}$ C. R. Wronski et al., in Proceedings of the 11th European Photovoltaic Solar Energy Conference and Exhibition, Montreux, Switzerland, 1992, edited by Leopoldo Guimaraes et al. (Harwood Academic, Chur, Switzerland, 1993), p. 72. 